\title{
PLAN DEVELOPMENT PROCESS AS A METHODOLOGY FOR CONTEMPORARY URBAN PLANNING
}

\author{
Marija Maruna* \\ University of Belgrade, Faculty of Architecture, Belgrade, Serbia \\ Vladimir Maruna \\ MD \& PROFY DOO, Belgrade, Serbia
}

The concept of the Plan Development Process is based on the possibility of application of the Unified Process methodology on urban planning process. Viewed as a way of organization of planning tasks, the urban planning process may be analyzed in the area of complex process management, particularly the area of software development management which gave rise to some of the most advanced methodologies for arrangement of tasks and standards pertaining to formal methodologies for definition and implementation of program solutions. Complex process management and urban planning face problems of similar complexity. The Unified Process methodology is one of the most successful methods for the organization of software development process. Through the application of the Unified Process methodology on urban planning process, the concept of plan development was formulated, as a methodology for contemporary planning process and the corresponding metamodel as the final level of abstraction bringing together all knowledge of a given domain.

Keywords: Process management, Urban planning methodology, Unified process

\section{INTRODUCTION}

This paper stemmed from the research exploring the possibilities for enhancement of urban planning methodology based on the knowledge and practice of the Object Oriented (OO) Modeling and Unified Process. The urban planning process, viewed as a way of organization of planning tasks, may be analyzed in the area of complex process management, particularly the area of software development management which gave rise to some of the most advanced methodologies for arranging tasks and responsibilities and standards pertaining to formal methodologies for definition and implementation of program solutions.

Contemporary process management is based on formal methods and comprehensive modeling has recently become one of the common ones [01]. Modeling as a method is one of the most successful approaches in identification, elaboration, collection, specification and presentation of a complex structure, dynamic and behavior or certain processes which is an excellent platform for collaboration, cooperation and communication between the involved parties [04]. Object oriented methodologies are emerging and currently are dominant in the IT since they can be easily used for any problem or solution domain as well as for an arbitrary complexity level/6/.Methodologies based on object oriented paradigm provide a simple and efficient transition from the highly conceptual level down to the practical and implementation level, that is, transition from analysis (problem) domain to design and implementation (solution) domain.

One of the most prominent, well-known and detailed software development methodologies covering the overall software development cycle is Unified Process (UP). The main advantage of the Unified Process is its strong orientation toward user functional and non-functional requirements with the aim to identify, understand and present motivation and objectives of the involved parties as well as the purpose of the particular activities within the process and the purpose of the process itself [14]. The Unified Process applies iterative and incremental approach that results in incremental advancement towards targeted goals and objectives. Iterative and incremental approach facilitates constant knowledge classification and integration as well as further enrichment of it through analysis, design and re- 
search, and in that way promotes and encourages knowledge dissemination and accumulation.

Problems of similar level of complexity, a large number of parameters in a heterogeneous and vast setting with a complex network of inter-relations, as well as the development of new techniques and tools are features common to both complex process management and urban planning [07]. Similarities between complex process management and urban planning are revealed by a comparative analysis of problem aspects within the two fields, such as: complexity and scope of problems and the need to address them in an interdisciplinary framework [03], the need for intensive communication between actors, specification of sub-topics, importance of the implementation phase, expressing problems in a formal way, documenting of data, transfer and accumulation of knowledge.

The initial steps in the research include describing and defining of the planning process, i.e. specification of its basic elements. Elements of the urban planning process are the basis for building an integrating development platform for IT tools in urban planning and further building of standards within the process, which will enhance communication between different actors, as well as among various tools and levels of abstraction $[13,15]$. The expected end result in the application of the Unified Process methodology on urban planning process management is the development of a new methodological approach in urban planning, the so called Plan Development Process and Urban Planning Meta-Model accumulating all the available knowledge in this area [09].

\section{THE CONCEPT OF THE PLAN DEVELOPMENT PROCESS}

The concept of the Plan Development Process is founded on positive experiences of the Unified Process methodology in complex processes management [16]. In the domain of IT, the progress of software development process, that is, the creation of clear directions for the development of software run parallel with the development of appropriate tools. Converse is true in the urban planning domain. Various tools have been developed for supporting individual tasks in the planning process; however, a unified platform for the organization of the urban planning process itself is missing. Such platform would also allow for the unified application of computing tools.
A unified urban planning platform, or, in other words, the Plan Development Process, would establish planning rules, that is, define a planning methodology suited to the logic of computer supported tools [08, 15].

Plan Development Process is induced by and founded on the software development process and object-oriented methodologies [09, 20], in general, and Unified Process, in particular sense. Methodology of the Unified Process is applied on the domain of urban planning to develop a new urban planning methodology which will address all elements of the domain and establish Plan Development Process together with Planning Meta-Model serving as an urban domain knowledge base. Plan Development Process together with Planning Meta-Model can and should be later used as a universal integrative and communication platform for all planning activities. Every particular plan would be developed with the implementation of elements of the Plan Development Process and with the instantiation of meta-model artifacts. In that sense every plan would be a particular instance of the Planning Meta-Model.

Development of the Plan Development Process methodology and the corresponding meta-model is iterative and incremental process which should run in parallel. The first step toward the meta-model is standardization that includes specification of methodology elements and domain language for communication, exchange and accumulation by using a formal language and techniques, in this case, UML, BPMN etc. Development of a meta-model would be incremental and iterative, starting from simple and small models and methodology elements to be later developed into a full-fledged meta-model and powerful methodology. The Urban Planning Meta-model would be then used as a knowledge base for urban planning [17]. Process of Plan Development Process development should be driven and constrained with the implementation of contemporary quality control methods that will be integrated with the development process to ensure consolidation and inclusion of all stakeholder requirements, with significant number of stakeholders, and different currently used quality management standards.

In general, the Planning Meta-Model demonstrates the possibility for ordering the whole set of activities and the organization of the ur- 
ban planning process through formal definition of who does what and when, and how certain goals are accomplished, that is, which actor is supposed to produce which artifact at a certain moment $[02,05]$.

\section{ELEMENTS OF THE PLAN DEVELOPMENT PROCESS}

The Unified Process identifies and specifies four phases of the software development process: Inception, Elaboration, Construction and Transition. Every phase aims to achieve a clearly specified set of goals and to produce a pre-defined set of artifacts. At the end of each particular phase a predefined set of evaluation criteria is verified and confirmed in order to decide whether to continue with the next phase or to stay in the current one. In every phase where iteration is executed, a new increment is produced and integrated with the current version, as a pre-version of the final solution, to be further developed through consequent releases and increments.

Following the principles of the Unified Process methodology, it is possible to define four groups of elements of the Plan Development Process: phases, workflows, actors and artifacts (products of planning activities). Author already presented first iteration of Plan Development process by detailed specification of first versions of phases, actors and artifacts in theirs previous papers. Consequently, this paper will give just an overview of listed element of Plan Development Process while the main focus will be on detailed presentation and specification of the first version of Plan Development Process workflows.

Phases - The Unified Process separates the phase of implementation and the phase of delivery, since it is possible to produce solutions independently, before their installation and use. Situation is somewhat different in urban planning so these activities cannot be divided into separate phases. Specifically, the concrete output of the planning process is not a tool or software which can be put to use, but rather, planned solutions which need to be continuously applied in a real setting. In software developments processes, system adjustment is performed in the delivery phase, that is, during use, while detection and correction of mistakes is a part of the final phase. On the other hand, planning process considers the problems as early as the initial phase, that is to say, starts from the demand to correct certain features of the urban environment, and consequently, in this respect, there is no delivery phase in urban planning process. Consequently, following the Unified Process logic, three phases of the planning process are suggested: inception, elaboration and implementation.More details on Plan Development Process Phases can be found at our previous work [17].

Workflows - Under the Unified Process activities are defined as a concrete set of tasks performed by actors in workflows which 1) imply clearly defined responsibilities of actors, 2) yield clearly defined results (set of artifacts) based on clearly defined input (another set of artifacts) and 3) present a working unit with firmly defined boundaries in relation to the planning project within which individuals are assigned tasks. It can also be defined as performing of operations assigned to actors [12].

Activities are mutually connected acts aimed at the development of an appropriate solution. The same activities can be repeated within different phases, and, where necessary, repeated in every phase. Activities are performed by assigned actors, following the sequence of performance of planning tasks and of the production of specific planning artifacts. Related activities within the Plan Development Process are usually called workflows. Following the Unified Process methodology, groups of related activities in the urban planning domain can be divided into the following workflows: Urban and Spatial Analysis, Requirement Analysis, Plan Formulation and Plan Execution and Revision.More detailed specification of workflows can be found below at "Starting models of the Plan Development Process - First iteration of the Planning Meta-Model - workflows and activities".

It is important to distinguish workflows from phases. Although certain workflows are better suited to certain phases, iterative-incremental approach implies the execution of all activities and workflows within each phase (Figure 1).

Phased execution of planning process activities simplifies the process of development of planned solution through separation of smaller working units - sequences, reducing the level of problem complexity and allowing for gradual arrival at the solution. 


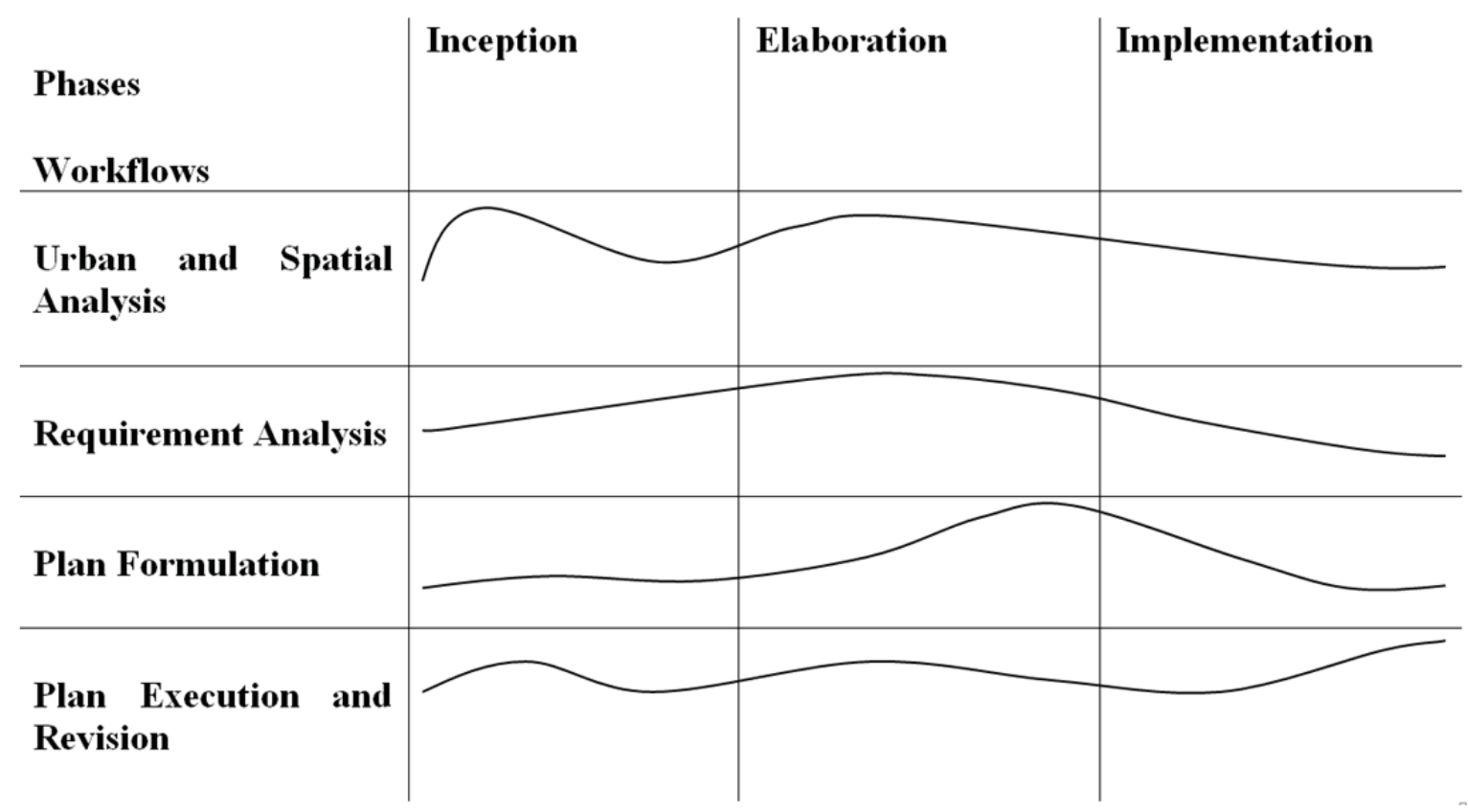

Figure 1: Life cycle of the Plan Development Process (done according to the life cycle of software development process, Jacobson, Booch and Rumbaugh, 1999)

Each phase is an independent entity with a concrete product - increment to which supplements and changes may be added as needed, contingent on the adjustments in other planning process phases. Phased execution of the planning process activities allows for a cyclical development of a planned solution, which is essential for ensuring flexibility, effectiveness, reliability, manageability and organization of dynamic processes such as the Plan Development Process.

Actors - In accordance with the main goal of software engineering - to deliver a software product satisfying determined business needs effectively and timely - the Unified Process defines which actor, at what time, and in what manner is to produce a specified artifact. An actor is a position in the process which can be allocated to an individual or a team, depending on responsibility and capability such as the performance of a specified activity and the development of a specified artifact [12].

Contemporary urban planning envisages broad participation of actors, i.e. stakeholders in the planning process, in line with their respective interests, capabilities, responsibilities and roles, equally in all phases of the process. Hopkins et al. recognize that participation of actors in contemporary planning process is determined by the following characteristics: interest, authority, power, right, technical capabilities, knowledge, responsibility, finances, and norms of behavior [10]. The degree to which actors participate in the planning process directly determines their respective roles relating to the use, production and management of artifacts, that is, products of the planning process. By the role in the planning process, in line with different capabilities, knowledge and understanding of the planning process, more specifically, the ability to take part in the production of planned solution, it is possible to distinguish four groups: politicians, investors, technical staff (experts) and the public [16]. More detailed analysis of the actors of the contemporary planning process is beyond the scope of this paper.

Artifacts - Artifacts, a term used to refer to every and any digital and non-digital "tangible" thing, are products of planning activities. Artifacts are created during the planning process as results of particular planning activities within a particular planning workflow in a particular planning phase by a particular actor as defined and specified within the Plan Development Process.Artifacts are products of the planning process, all input and output information, documents, drawings, calculation, alternatives, results or analyses, presentation of problems etc. Classification and systematization of artifacts is partially, in that sense, inducted and shaped by classification and systematization of activities and workflows. Artifacts are grouped together into models in accordance with the corresponding workflow that produces them. Consequently, every workflow has a corresponding artifacts. Every artifact is established once and then improved over a period of time in accordance with the iterative and 
incremental principle within a particular workflow and phase. Although artifacts can and will be changed not only in the workflow where they belong but also in other workflows, we would propose to use this workflow-based classification and divide artifacts in the following groups:

- Urban and Spatial Analysis artifacts

- Requirement Analysis artifacts

- Plan Formulation artifacts and

- Plan Execution and Revision artifacts.

Artifacts grouped into Urban and Spatial Analysis group include a set of input information used to start the whole planning process. This is mainly information about the current urban and spatial conditions, problems and constraints, current and expected trends, available resources together with the information (artifacts) inherited from the plans from upper level of planning as particular set of guidance rules and upper level requirements and constraints.

Requirement Analysis artifacts include all that is related to the requirements, motivation and concerns of involved parties. Different background, vocation, level of involvement and similar will define the scope and nature of the requirements imposed on the process by certain actors.

Plan Formulation artifacts group includes all products of the planning process which are directly connected to the plan design and assessment and selection of alternatives, starting from initial sketches and ideas, through the development and design of various options and their assessment, to the final decision and selection of a particular alternative. Plans are particular frameworks used to identify, elaborate and present consensus about the future development of a certain aspect of a particular domain which is developed down to the sequence of actions, with required and appropriate financing scheme, to implement and manage required and agreed changes.

Execution and Revision artifacts include products created during the implementation of the previously established planned solution as well as artifacts created during the further elaboration of this solution into more specific and detailed implementation steps.

\section{STARTING MODELS OF THE PLAN DEVELOPMENT PROCESS - FIRST ITERATION OF THE PLANNING META-MODEL - WORKFLOWS AND ACTIVITIES}

Here is presented the first versionof the Planning Meta-Model as part of the Plan Development Process, which is based on the Unified Process as explained above.

We have previously defined workflows and activities, as well as artifacts and actors in their first version [18, 19]. Further in the text we explain how activities are organized within a particular workflow and how particular artifacts are used and by what activity. Flow diagrams are used to present the sequence of processes and activity execution, all possible transitions between them, conditions to be checked before transitions can be executed, point of decision with all possible outcomes, points of branching of activities to parallel flows, points of joining further decomposition of processes containing other processes, usage of resources and other artifacts etc., all specified by using the latest process notation named BPMN. The presented diagrams in this sense, together with the elements presented on them and their specification will form the Plan Development Process workflow meta-model.

The workflows proposed previously are Urban and Spatial Analysis, Requirements Analysis, Plan Formulation and Execution and Revision. Accordingly, we will present five different diagrams, where the fifth one presents the overall process $[18,19]$.

One of the targeted and important characteristics and advantages of the Plan Development Process is incremental and iterative approach that has to be applied at every level of the proposed methodology but which is especially important to apply it at the level of the whole process. When this principle is applied, the Plan Development Process is organized as one iterative and cyclical process which includes and organizes the constituent four workflows in one complex orchestration without any actual start and stop and with several feedback transitions (Figure 2).

The presented workflow overview explains the organization and relationships between the four workflows for the overall Plan Development Process (PDP). The figure obviously presents two main aspects of PDP, that is, its linearity to advance from Urban and Spatial Analysis toward 
Execution and Revision, if and when it is applicable, and its cyclical pattern to adjust the over- all progress in each and every process phase, if and when it is applicable.

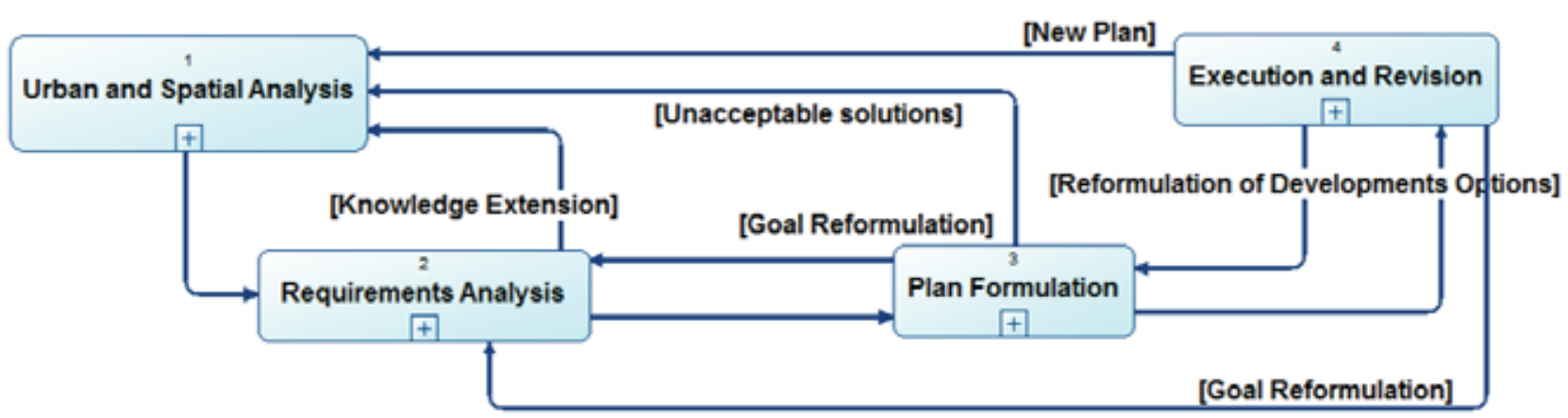

Figure 2: Plan Development Process - workflow model

The presented process therefore may have a linear execution path starting from Urban and Spatial Analysis, followed by Requirement Analysis and Plan Formulation and ending with Execution and Revision. Direct path of execution, although not most likely, will hold the focus of overall activity straight and it is goal oriented. Cyclical pattern will, on the other side, facilitate and provide opportunity to adjust to the changes and insights arising during the process itself. Every feedback is endorsed with the condition (change) that will occur and triggers transition back to one of the previous workflows. After that, Urban and Spatial Analysis process will continue with Requirement Analysis, but if extension of the knowledge on any aspect of the treated urban system is needed the process will return execution back to Urban and Spatial Analysis. If in Plan Formulation one discovers that something is not specified properly in Plan Goals, the process would have to return back to Requirement Analysis to see what is missing, or if one finds that the overall solution is not acceptable, because some of the basic assumptions are wrong or similar, the process can be returned even to the Urban and Spatial Analysis. The last step is Plan Execution and Revision where the planned solution is implemented and executed in a real environment. Although this workflow contains a built-in mechanism and capabilities for adaptation during the implementation, the required changes and revision can be wider and broader then it is manageable in the Execution and Revision when several possible choices are available. When new plan options and alternatives are needed, the process will return to the Plan Formulation, if and when one of the Goals should be revised, the process will return to the Requirement Analysis and if a completely new plan is required, because required corrections are too wide and broad, the process can even return back to Urban and Spatial Analysis and in that way start a whole new cycle and new iteration.

Before we present the actual workflows, we will provide a short explanation of the three different "usage" relationships that are used on the diagrams to note how a certain process uses certain artifacts all presented below on Figure 3.

All diagrams below consist of different activities and different artifacts. Artifacts, presented on the diagram, can be created and originated in the current workflow which is presented by a dotted line, like for example between Resources and Resource Assessment on Figure 4. Artifacts may also be only used in the current workflow but their origin can be either upstream or downstream in the overall process. If an artifact is produced after the current workflow, downstream, it can be used in the current workflow either after the feedback or in the next iteration. Downstream usage of an artifact is presented by a full line like for like for example between Conflicts and Problem Identification on Figure 4. Upstream usage is presented by two-dot-line line like for example between Trends and Forecasting on Figure 5 below. The same notation is used on all other diagrams.

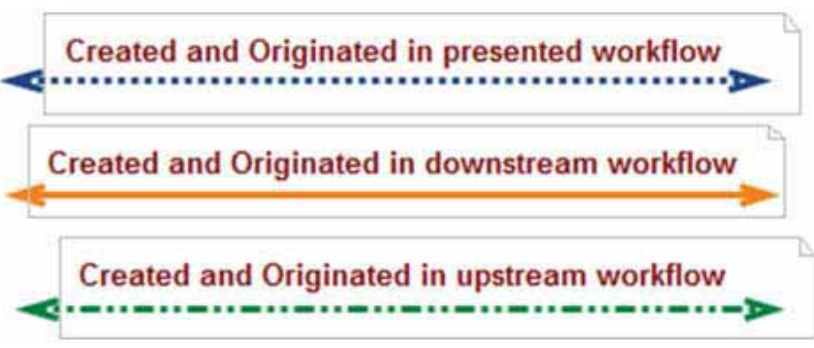

Figure 3: Explanation of Lines

Activities in this workflow aims to identify, elaborate, specify and present the current status of the treated urban system through its important 
aspects including spatial, social, economic, legislative and institutional context. Urban and Spatial Analysis should recognize and specify all basic and important problems in the treated urban system by discovering, understanding and documenting trends and assess problems and their seriousness and extent. It is also important to evaluate the overall capacity, availability and sustainability of resources i.e. capability of the area. Elements identified in this workflow are not only of urban or spatial nature.

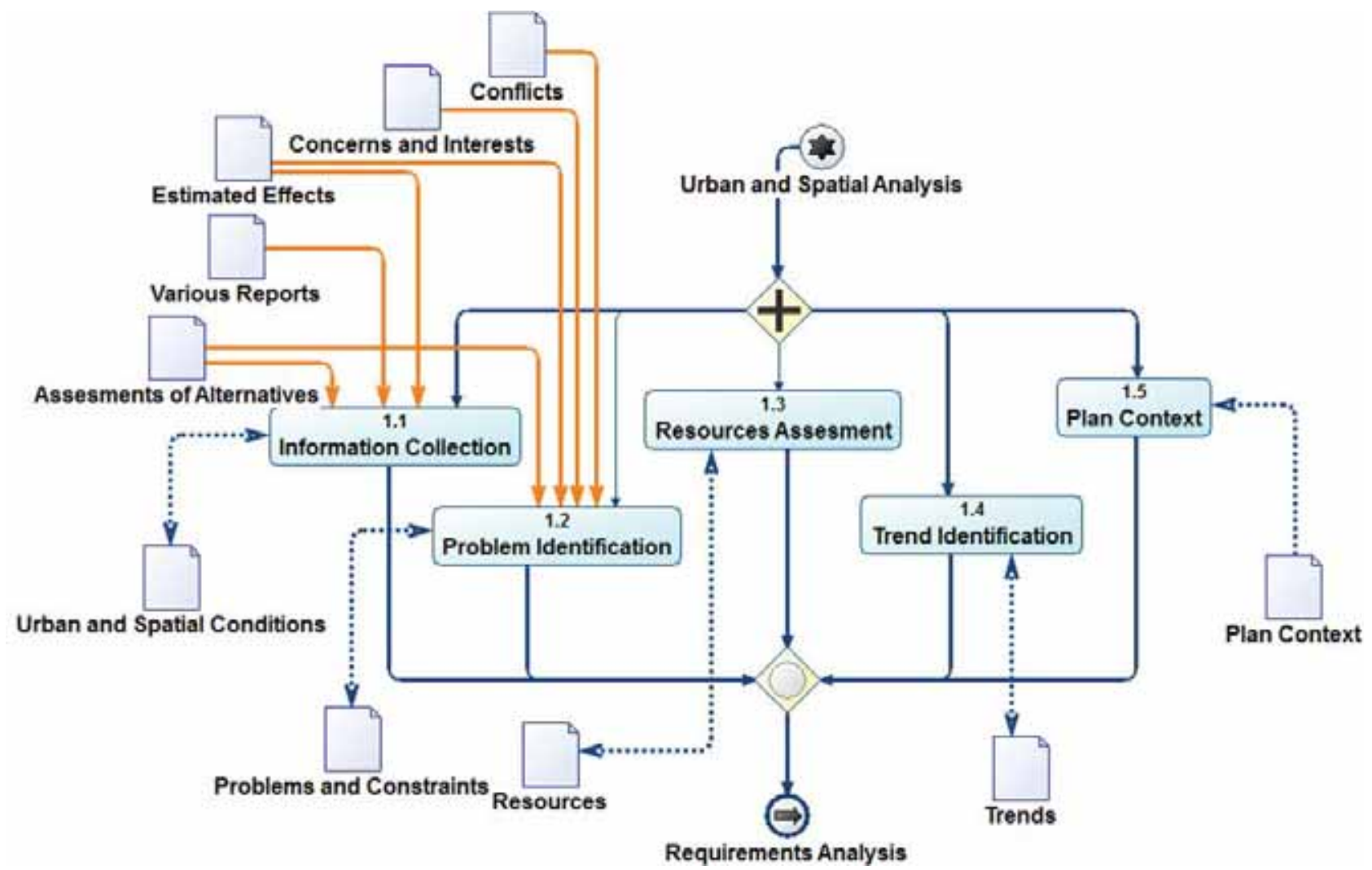

Figure 4: Urban and Spatial Analysis

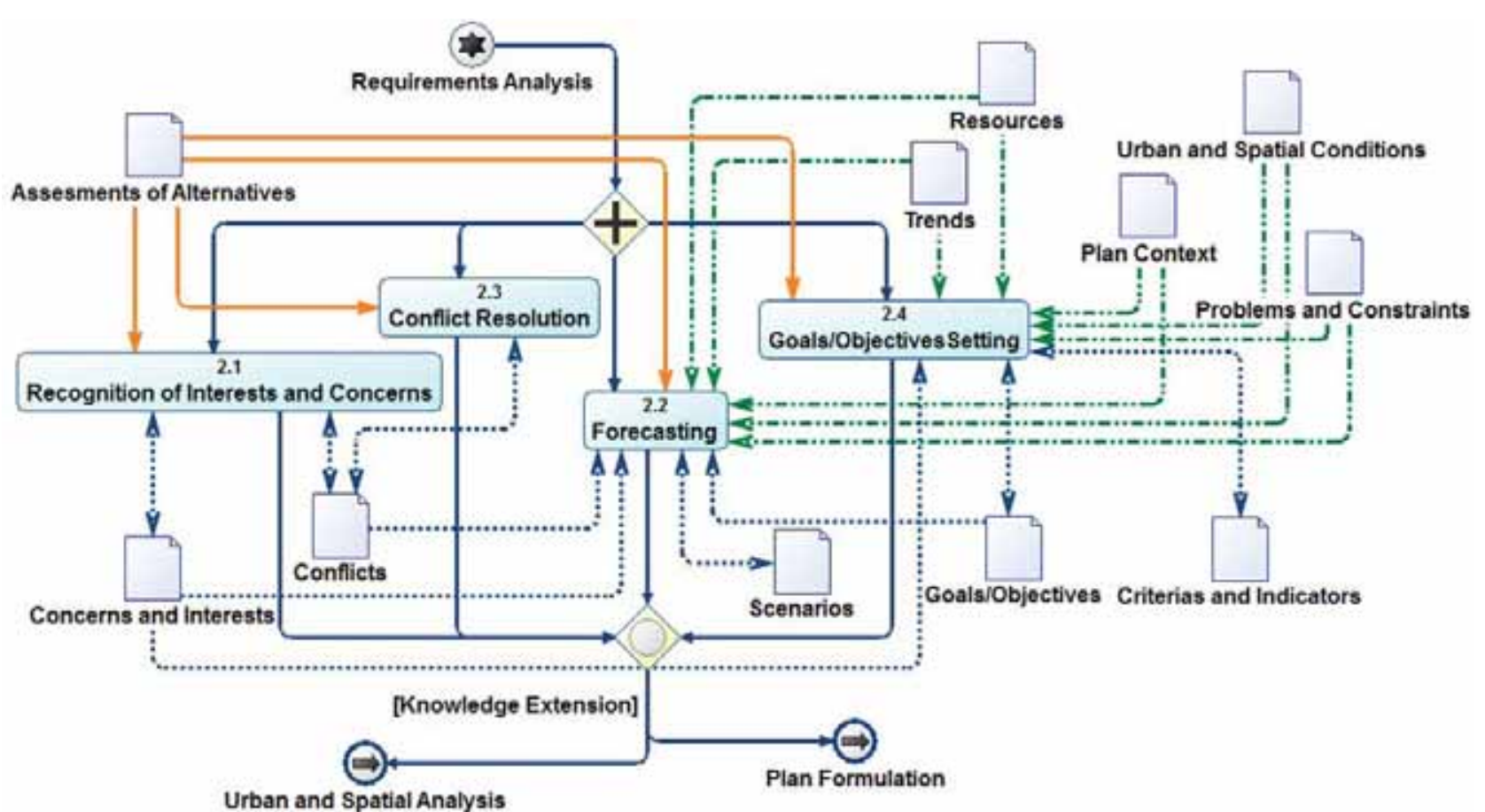

Figure 5: Requirement Analysis 
Urban and Spatial Analysis contains five different activities named Information Collection, Problem Identification, Trend Identification, Resources Assessment and Plan Context, which may be executed in parallel, while the workflow ends when all of the activities are finished at least in first iteration. Information Collection tries to collect, transform, save and present all important details related to the treated urban system in the form of Urban and Spatial Conditions by using Artifacts, Conflicts, Interests, Effect and Reports, developed in either Requirement Analysis or Plan Formulation, which may happen if feedback is required or new iteration is started.

Problem Identification tries to establish the full list of problems by using also artifacts produced downstream in Requirement Analysis or Plan Formulation. Resource Assessment tries to evaluate capacity, availability and sustainability of resources i.e. capability of the area and to present this within Resources. At the end, Plan Context tries to identify and present constraints of higher plans and regulations and save this as Legal Constraints, together with requirements and constraints that are immanent to the treated urban and spatial domain.

Requirement Analysis establishes the full collection of all actors' requirements, including citizens, experts, government representatives and institutions, investors and similar, and identifies plan constraints and issues to be addressed in the subsequent workflows and plan phases. Requirements and constraints induced by the higher level regulations and policies should be included to create the output which will be compatible with them. Follows development and determination of development directions and theirs reconciliation toward user requirements. At the same time are identified and registered group of conflicts of interests between different actors together with the platform for reconciliation of conflicts and establishment of mutual agreement. This includes identification and specification of relations between requirements of different actors recognizing the level of compatibility (conflicted, compatible or inert). The objective is to reconcile, exchange and assess this usually conflicted set of demands and reach a consensus acceptable for all by using techniques such as negotiation, mediation, facilitation etc., known from conflict management theory and practice, but also simulation, visualization and 3D modeling of certain aspects of the planning artifacts.
This minimal set of reconciled Actor's requirements is first used to establish and apply planning priorities and later to promote those prioritized requirements as planning goals and objectives. Defined set of Goals is accompanied with a set of criteria used for operationalization of individual Goals and later in the scope of the last workflow for evaluation of the solution success.

Requirement Analysis contains four different activities named Interest Recognition, Conflict Resolution, Forecasting and Goal/Objective Setting, which may be executed in parallel, while the workflow ends when all the activities are finished at least in first iteration. At the end of this workflow, the planning process may continue with the next workflow, Plan Formulation, if the result of this workflow is adequate, or it may be directed back (feedback) to the Urban and Spatial Analysis if additional knowledge is required.

Recognition of Interests and Concerns establishes full and complete list of Actor's motives and interests and may use Assessments of Alternatives produced in Plan Formulation either in the previous iteration or in this iteration, when feedback is also requested, to identify and specify interests of all actors and to map a complex network of possible conflicts between them.

Here the process recommends a reconciliation of this usually conflicted set of demands in order to reach and establish consensus acceptable for all by using Conflict Resolution activity. The analysis of the requirements and interests would promote and establish the set of Objectives/Goals accompanied by the list of Criteria and Indicators to be used later in Plan Formulation to develop and assess Alternatives and Options. At the end, Forecasting would aim to establish Scenarios, to explain possible Effects of the plan, based on the results of Urban and Spatial Analysis, by using Artifacts produced there, and on the basis of Alternatives produced in Plan Formulation.

Plan Formulationworkflow (Figure 6) designs the solution capable of resolving listed set of issues, fulfill defined requirements and achieve set Goals/Objective, identified previously, in compliance with the higher level recommendations and regulations and using available resources of any kind, such as available expertise, time, financial support, technology etc. Plan Formulation workflow is used to establish planned solution with all required details through development and assessment of planning alternatives. Every alterna- 
tive is actually draft developed down to the level that is adequate to understand and evaluate of particular concept and derived planned solution what is based on the result of previous workflow. Assessment of alternatives includes identification and elaboration of possible problems, related to this alternative, and uncertainty risk analysis.
Selected set of alternatives are then developed further into planned solution with properly set priorities and phased implementation.Activities in this workflow are mostly conducted by experts of different kinds, and include forecasting, assessment, analysis, optimization and evaluation of different development options and alternatives.

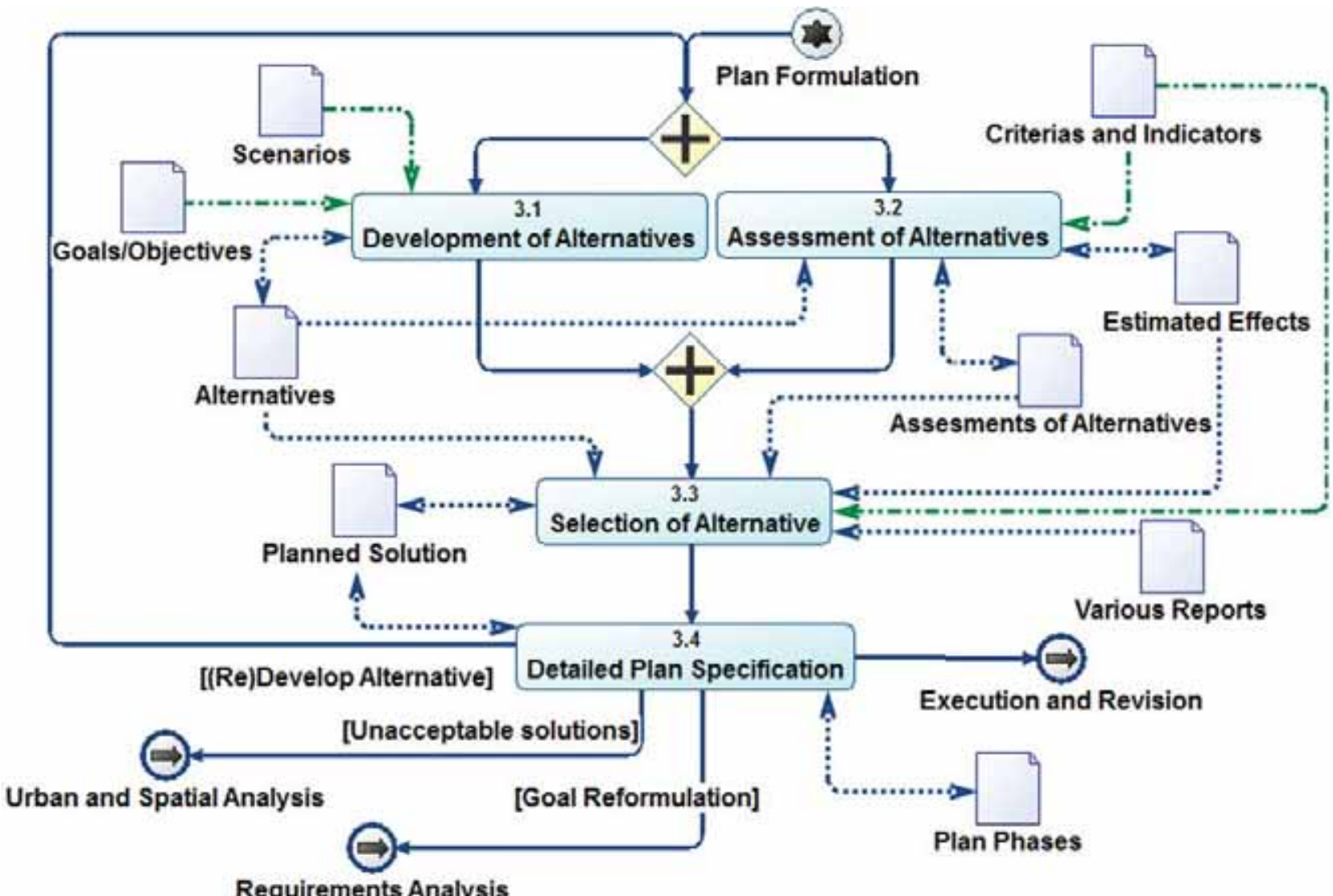

Figure 6: Plan Formulation

Plan Formulation contains four different activities named Development of Options, Assessment of Options, Preferred Option Selection and Detailed Plan Specification, but the organization in this workflow is slightly different than in the previous two. The first two activities, meant to develop and asses plan Alternatives, are executed in parallel but Plan Formulation may continue only when and if both of them are finished. Only then we can select one or more preferable Alternatives indirectly or directly on the basis of previously defined Goals, Alternatives, Conflicts, Interests, Resources, Capabilities etc. Finally, this selected set of options is promoted into a full-fledged planwith detailed plan specification and detailed development of plan phases. During the development of plan details it may happen that particular Alternative needs to be redeveloped or asses again when flow is redirected back to the part of the workflow responsible for development and assessment of Alternatives. Moreover, during the development of the detailed specification of a plan one may discover major flaws, inadequate assumptions, undeveloped or missing elements etc. and in this case one would flag an unacceptable solution and the overall process would have to be redirected back (feedback) to Urban and Spatial Analysis or, if the problem is related to the selected set of Goals, back to Requirement Analysis. If the developed solution does not trigger any of the previous actions, the overall process would continue with Execution and Revision.

Execution and Revisionworkflow (Figure 7) uses the previously adopted plan and the corresponding artifacts to define and prioritize the list of activities that need to be executed in the form of one or more tools or instruments of implementa- 
tion. Plan implementation will also include procedures to be followed, executed and controlled during its realization. The implementation will have to include a constant monitoring of results and effects of plan execution and implementation against the predefined indicators and an assessment of plan impact on the ambient. At the end, the implementation will have to evaluate whether the revision of the plan or a tool is necessary, and whether the assessed impact is adequate or not, in which case the plan has to be revised and/or the implementation tools and instruments have to be corrected.

Execution and Revision contains four different activities named Development of Implementation Tools, Assessment of Effects, Monitoring of Implementation and Plan Adjustment, but in this orchestration the organization is slightly different than in the previous three. The first and mandatory action at the beginning of Plan Implementation and Revision is the development of Implementation Tools containing a prioritized list of activities and responsibilities, implementation procedures, legislation elements etc. Only after the Development of Implementation Tools is finished actual implementation of planned solution in real ambient may start. During this implementation two parallel processes are activated named Monitoring of Implementation, to supervise and monitor plan execution and implementation, and Assessment of Effects, to evaluate what are the effect on the urban ambient. Those processes are executed independently of each other. Any of those two activities may raise and request verification of the plan if inadequate results are perceived, in which case Plan Adjustment is activated. Even where no major flaws are triggered, the process periodically Checks Implementation status and if inadequate results are perceived Plan Adjustment is activated. If changes are not related to the plan but to the implementation tools and instruments, the process will re-develop Implementation Tools and continue with Implementation and Revision. If changes are related to the plan but are not substantial and revision may be contained within the boundariesof correction of the plan, the process will stay in Implementation and Revision and Corrected Plan will be implemented further on. Only if required and necessary changes are exceeding the boundaries of the existing plan, the process will continue with one of the previous workflows (feedback) depending on level of changes. If redevelopment of alternatives is required, the process will continue with Plan Formulation, if goals have to be redesigned and reformulated, the process will continue with Requirements Analysis and, ultimately, if changes are substantial and exceed the solution, and new plan is the only option, in which case the process will start new iteration with Urban and Spatial Analysis.

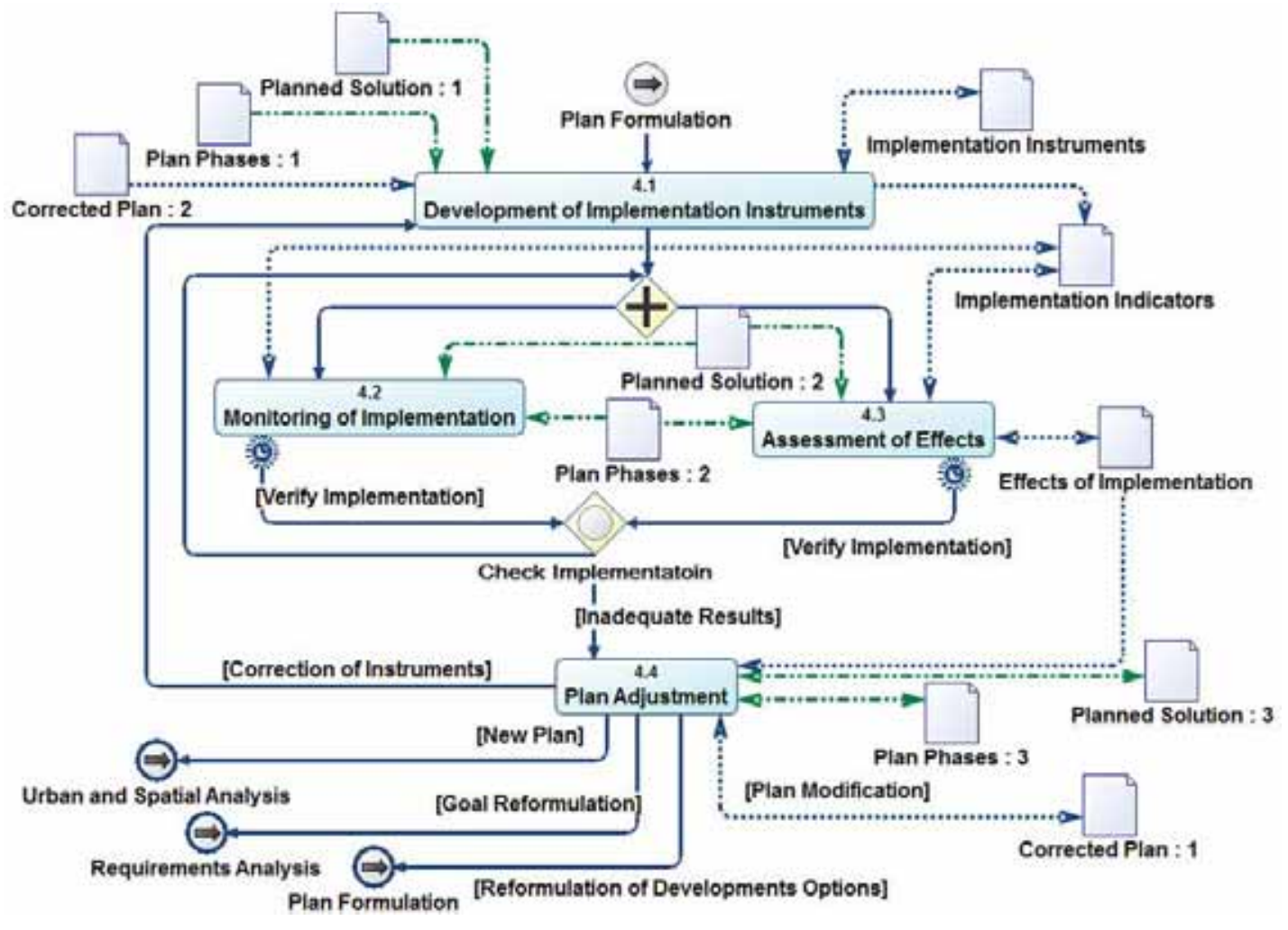

Figure 7: Execution and Revision 


\section{CONCLUSION}

The starting models of the Plan Development Process, although presented in a simplified manner, demonstrate advantages and possible benefits from such methodological approach. Creation of small models and elements of the methodology forms a foundation for iterative advancement towards a complex, powerful and useful methodology. The first iteration of the Planning MetaModel demonstrates the possibility for ordering the whole set of activities and the organization of the urban planning process through formal definition of who does what and when, and how certain goals are accomplished, that is, which actor is supposed to produce which artifact at a certain moment. Foundation has been laid for better quality management of the planning process through bringing in order activities of the planning team, directing team tasks and specifying types of artifacts to be produced. Through the implementation of the first iteration of the Planning Meta-Model it has been confirmed that models accurately present the components of the planning process and define the procedure for the development of the planned solution.

A visual representation of the planning process by a model, that is, the transformation of the textual format into a linked graph, is a linguistic transformation of a kind. Formal representation of the planning process by model improves the intelligibility of procedures and allows for easier communication between participants at different levels and in different domains of knowledge and skills. Formal representation of the planning procedure, or more precisely, the creation of formal methodology is a precondition for the development of standards and creation of semantic and syntactic rules, that is, the development of a universal language solving the problem of communication between various actors.

Planning process models also form a database of a kind, that is, allow for straightforward monitoring of information produced during the process of solution development. In this manner planning process model ensures recording of data, since each model in itself is a record of things done, decisions taken and choices made. Models replace paper documentation and thus simplify and upgrade exchange of information and communication, make possible continuous advancement of the content through iterations, simplify data storage and allow for incremental growth of knowledge.By crafting Plan Development Process models, with the aid of computer notations, better and more direct communication is established with IT experts, which is one of the main preconditions for the development of advanced and more effective applicative solutions to support urban planning. Further development of the planning process along with the supporting models will certainly contribute to the advancement of such cooperation, which should upgrade the level of IT support to urban planning activities.

\section{REFERENCES}

1) Abu Gaben, M., Krčevinac, S., Vujošević, M. (2007) Modelujući sistemi u optimizaciji, Journal of Applied Engineering Science (Istraživanja i projektovanja za privredu), No.18, pp. 37-47

2) Andersen, D. F., Bryson, J. M., Richardson, G. P., Ackermann, F., Eden, C., Finn, C. B., (2006) Integrating Modes of Systems Thinking into Strategic Planning Education and Practice,Journal of Public Affairs Education, 12(3), 265-293.

3) Batty, M., Torrens, P. M., (2001) Modeling Complexity: the limits to prediction,CASA Working Paper Series, 36, London: Centre for Advanced Spatial Analysis University College.

4) Brans, J. P., (2004) The management of the future Ethics in OR: Respect, multicriteria management, happiness,European Journal of Operational Research,153, 466-467.

5) Galle, P., (1991) Alexander Patterns for Design Computing: Atoms of Conceptual Structure, Environment and Planning B: Planning and Design,18(3), 327-346.

6) Gamma, E., Helm, R., Johnson, R., Vlissides, J., (1995) Design Patterns - Elements of Reusable Object - Oriented Software, Addison - Wesley.

7) Hamilton, A., Wang, H., Tanyer, A. M., Arayici, Y., Yhang, X., Song, Y., (2005) Urban Information Model for City Planning,ITcon, 10, 55-67.

8) Harris, B., Batty, M., (2001)Locational Models, Geographic Information, and Planning Support Systems, Planning SupportSystems, Redlands, California: ESRI Press, 25-58.

9) Hopkins, D. L., (2001) Structure of a Planning Support System for Urban Development, Planning Support Systems, Redlands, California: ESRI Press, 81-98. 
10) Hopkins, D. L., Kaza, N., Pallathucheril, V. G., (2005) Representing urban development plans and regulations as data: a planning data model, Environment and Planning B: Planning and Design,32(4), 597-614.

11) Hopkins, D. L., Zapata, M. A., (2007) Engaging the Future, Forecasts, Scenarios, Plans, and Projects, Cambridge, Massachusetts: Lincoln Institute of Land Policy.

12) Jacobson, I., Booch, G., Rumbaugh, J., (1999) The Unified Software Development Process, Addison - Wesley.

13) Klosterman, R., (1999) New perspectives on planning support systems,Environment and Planning B: Planning and Design, 26(3), 317-320.

14) Larman, C., (2002) Applying UML and Patterns, An Introduction to Object-Oriented Analysis and Design and the Unified Process, Prentice Hall PTR.

15) Laurini, R., (2001) Information systems for urban planning - A hypermedia co-operative approach, London and New York: Taylor\& Francis.

16) Maruna, M., Maruna, V., (2005) Elements of Urban Planning Methodology Based on Rational Unified Process, Proceedings of The 9th International Symposium on CUPUM 05, University College London, 29 June-01 July.
17) Maruna, V., Maruna, M., (2005) Prospective Enhancement of Urban Planning Methodology Based on $\mathrm{OO}$ Modeling and Rational Unified Process, Proceedings of The 10th International Symposium on CORP 2005\&GEOMULTIMEDIA 05, TU Wien and Multumediaplan.at, Wien, 22-25 February.

18) Maruna, V., Maruna, M., (2007) Preliminary OO Specification of Planning Artifacts, Urban Planning Meta-Model, Proceedings of The 10th International Symposium on CUPUM 07, University Sao Karlos, Sao Paolo, Iguassu Falls, Brazil, 11-13 July.

19) Maruna,M.,Maruna, V., (2009) Toward Planning Development Process: Workflows in Urban Planning Meta-Model, Proceedings of The 11th International Symposium on CUPUM 09, University of Hong Kong, 16-18 June.

20) Shiode, N., (2000) Urban Planning, Information Technology, and Cyberspace,Journal of Urban Technology, 7(2), 105-126.

Paper sent to revision: 17.04.2013.

Paper ready for publication: 16.05.2013. 